Orion Pharma, Novartis, Pfizer, I. Olsen: None declared, N. Bolstad: None declared, K. Jørgensen Consultant for: Celltrion, Intercept, AbbVie, Tillott, M. Lorentzen: None declared, C. Mørk Consultant for: Cellgene, AbbVie, Galderma Nordic, ACOhud, Novartis, LEOPharma, J. Jahnsen Consultant for: Celltrion, Orion Pharma, Pfizer, MSD, AbbVie, Takeda, Napp Pharma, AstroPharma, E. Haavardsholm Consultant for: AbbVie, UCB, Pfizer, MSD, Roche, T. Kvien Consultant for: Biogen, BMS, Boehringer Ingelheim, Celltrion, Eli Lily, Epirus, Hospira, Merck-Serono, Novartis, Orion Pharma, Pfizer, Sandoz, UCB DOI: 10.1136/annrheumdis-2017-eular.4438

\section{THU0355 INFLUENCE OF ADALIMUMAB AND SULFASALAZINE ON STRUCTURE OF HYALINE CARTILAGE OF HIP JOINTS IN PATIENTS WITH ANKYLOSING SPONDILITIS}

A. Petrov, H. Zayaeva, A. Petrov. Medical Academy named after Georgievsky, Crimean Federal university named after V.I. Vernadsky, Simferopol, Russian Federation

Background: The impairment of hip joints has significant prognostic value on functional status of patients with Ankylosing spondylitis (AS) [1]. One of possible yearly marker of hip joints structure damage in patients with AS may be changing in volume of hyaline cartilage [2]. But clinical significance of this marker in monitoring of hip joint structure changes during treatment is underinvestigated.

Objectives: To investigate changes in width of hyaline cartilage of hip joints in patients with AS under treatment with sulfasalazine and adalimumab during 12 month.

Methods: The 53 patients with AS (42 male, 9 female, average age is 37.6 years old, duration of disease is $14-152$ month) were included into study. All patients were treated by NSAIDs and sulfasalazine ( $2 \mathrm{~g}$ per day) at least 3 month before study. In treatment of 27 patients (1st group) was added adalimumab (40 $\mathrm{mg}$ subcutaneously every 2 weeks), other 26 patients (2nd group) were left on previous treatment regime. Patients were observed during 12 months of treatment including measurements of pain visual analog scale (VAS) in hip movements, maximal distance between ankles, pelvic X-ray and sonography of hip joints by 10-18 MHz probe. BASRI-Hips index was applied for radiographic estimation of structural damage of hip joints [3]. During sonography width of hip joint capsule and hyaline cartilage were measured. The Mann-Whitney-U test was used for comparison of changes in clinical and sonographic data between two groups of patients.

Results: After 12 month treatment period in patients of 1 st group in comparison with patients of 2nd group more significant decrease of pain VAS during hip movements (on 27.3 [18.8; 32.5] mm vs $4.7[0.5 ; 9.6] \mathrm{mm}, \mathrm{p}<0,01$ ), increase of maximal distance between ankles (on 124.3 [92.3;145.6] $\mathrm{mm}$ vs 3.5 [1.2; 6.5] $\mathrm{mm}, \mathrm{p}<0,05$ ) and decrease of joint capsule width (on $2.4[1.0 ; 3.6] \mathrm{mm}$ vs 0.4 [0.0;1.1] mm, $p<0,05)$ had been determined. In patients of 1st group width of hyalinic cartilage had been increased on $0.15[0.4 ; 0.22] \mathrm{mm}$, while in patients of 2 nd group width of hyalinic cartilage had been decreased on $0.8[0.0 ; 1.4]$ $\mathrm{mm}(p<0,05)$. These cartilage changes were accompanied by decrease of mean BASRI-Hips index on 1 point in 2nd group and absence of changes of BASRI-Hips index in 1st group. The correlation between changes in width of hip hyalinic cartilage and pain VAS during movement in hip joints $(r=-0.52[0.38 ; 061])$ and maximal distance between ankles $(r=+0.47[0.32 ; 0.60])$ had been revealed.

Conclusions: Treatment with adalimumab leads to decrease of clinical and sonographic signs of coxitis and improvement of hyaline cartilage structure. The increase of width of hip hyalinic cartilage correlates with clinical effect of treatment of coxitis in patients with AS. More prolonged observation is needed for analysis of correlation between changes in hyaline cartilage structure and radiographic progression of hip joints damage in AS.

References:

[1] Baraliakos X., Braun J. Hip involvement in ankylosing spondylitis: what is the verdict? Rheumatology 2009;49(1):3-4.

[2] Maksymowych W.P. Ankylosing spondylitis - at the interface of bone and cartilage. J Rheumatol 2000;27(10):2295-301.

[3] MacKay K, Brophy S, Mack C, et al. The development and validation of a radiographic grading system for the hip in ankylosing spondylitis: the bath ankylosing spondylitis radiology hip index. J Rheumatol. 2000;27:2866-72.

Disclosure of Interest: None declared

DOI: 10.1136/annrheumdis-2017-eular.1672

\section{THU0356 THE RANK OF ADMINISTRATION AND NOT THE MOLECULE HAS AN IMPACT ON THE ANTI TNF TREATMENT RESPONSE IN AXIAL SPA}

A. Moltó, A. Etcheto, L. Gossec, S. Perrot, N. Boudersa, P. Claudepierre, N. Roux, F. Berenbaum, A. Martin, L. Sparsa, P. Coquerelle, M. Soubrier, M. Dougados. Predict-SpA Study Group, Paris, France

Background: Five different sub-cutaneous TNF alpha blockers (TNFb) are available for the treatment of NSAID-refractory and active axial spondyloarthritis (axSpA). The efficacy of these drugs in axSpA has been well reported in several RCT and real-life studies separately or as a group, but only very few head-to-head studies have compared each molecule's treatment effect in axSpA. Also, some data suggest that TNFb treatment effect in patients failing to a first TNFb is poorer than in TNFb-naïve patients, but some other suggest that response to a second TNFb might be comparable.

Objectives: to describe TNFb prescription and treatment response of each TNFb, and to compare treatment response in TNFb- naïve/not naïve axSpA patients in a real-life setting.

Methods: Prospective, multicenter, usual care study with 2 visits (baseline and 12 weeks after TNFb initiation, Predict-SpA study, ClinicalTrials.gov: NCT03039088)). Patients: axSpA patients (diagnosis according to treating rheumatologist) initiating a TNFb. Data collection: Patients and disease characteristics at baseline. Previous exposure to TNFb was collected. Disease activity and function measures were collected at both visits. The choice of the TNFb to be started during the trial was at the treating rheumatologist's discretion. Statistical analysis: effectiveness was defined by the BASDAI50 response. Non-responder imputation and baseline observation carried forward imputation (for binary and continuous outcome variables, respectively) were performed for patients who discontinued the TNFb treatment between baseline and the follow-up visit.

Results: Among the 527 patients enrolled in the study, 508 patients were included in the analysis (1 patients was excluded due to missing data on all disease activity measures at baseline, and 18 patients were excluded due loss of follow-up between baseline and the follow-up visit). Mean age was $41.4( \pm 11.6), 237$ (47\%) were women, with a $6.1+8.5$ mean disease duration $377 / 508(74 \%)$ were TNFb-naïve while $66(13 \%), 35(7 \%), 19(3.7 \%), 8(2 \%)$ and $3(1 \%)$ had previously received, 1,2, 3, 4 and $>4 \mathrm{TNFb}$, respectively.

In the whole study population the most frequently prescribed TNFb was etanercept $(197(39 \%))$, followed by adalimumab $(131(26 \%))$, golimumab (88 $(17 \%))$, infliximab $53(10 \%)$ and certolizumab (39 (8\%)); the same order was observed in TNFb naïve patients; however, in patients previously exposed to TNFb, the most frequently prescribed TNFb was infliximab (31 (24\%)) followed by golimumab $(29(22 \%))$, adalimumab $(29(22 \%))$, certolizumab $(22(17 \%))$ and etanercept $(20(15 \%))$.

BASDAI50 responses were comparable for all TNFb molecules: 52\%, 52\%, $48 \%, 53 \%$ and $49 \%$ for etanercept, adalimumab, golimumab, infliximab and certolizumab, respectively.

BASDAI 50 response was comparable in patients TNFb-naïve and patients previously exposed to only 1 previous TNFb $(53 \%$ vs. $52 \%$, for naĩve vs. nonnaïve patients) but significantly decreased after (i.e. $37 \%$ and $36 . \%$ for patients previously exposed to 2 and to 3 or more $\mathrm{TNFb}$ )

Conclusions: this study suggests that the TNFb treatment response is similar across the different available molecules but this treatment response decreased in case of a previous use of two TNFb.

Acknowledgements: This study was conducted thanks to an unrestricted grant from MSD

Disclosure of Interest: None declared

DOI: 10.1136/annrheumdis-2017-eular.2678

\section{THU0357 NO SPECIFIC IMPACT OF CONCOMITANT FIBROMYALGIA ON THE ANTI TNF TREATMENT EFFECT IN AXIAL SPONDYLOARTHRITIS PATIENTS WITHOUT RADIOGRAPHIC STRUCTURAL DAMAGE OR OBJECTIVE SIGNS OF INFLAMMATION}

A. Moltó, A. Etcheto, L. Gossec, S. Perrot, N. Boudersa, P. Claudepierre, N. Roux, F. Berenbaum, A. Martin, L. Sparsa, P. Coquerelle, M. Soubrier, M. Dougados. Predict-SpA Study Group, Paris, France

Background: Currently, the European Medicine Agency (EMA) is recommending the use of anti-TNF in case of active NSAID refractory axial (ax) spondyloarthritis $(\mathrm{SpA})$ only in the presence of either structural radiographic damage of the sacroiliac Joints (SIJ) or objective signs of inflammation (either elevated CRP or inflammation at MRI-SIJ) probably mainly because of the fear of a concomitant fibromyalgia (FM).

Objectives: To evaluate the interaction of a concomitant FM on the anti-TNF response with regard to the application of the EMA recommendation

Methods: Study design: Prospective, multicenter, longitudinal (12 weeks) open trial (Predict-SpA, ClinicalTrials.gov: NCT03039088). Patients (pts): axSpA requiring an anti-TNF. Data collected: At baseline, 1/Radiographic SIJ damage 2/ MRI-SIJ inflammation 3/ Elevated CRP 4/ FM according to the FiRST (Fibromyalgia Rapid Screen Test) questionnaire. At baseline and after 12 weeks of therapy: BASDAI. Statistical analysis: Non Responder Imputation (NRI), Baseline Carried Forward Technic (BOCF) in case of missing data. Interaction test between the presence of FM and the fulfillment of the EMA recommendation (defined by the presence of either structural radiographic damage of the SIJ or objective signs of inflammation (either elevated CRP or inflammation at MRI-SIJ)) on the anti TNF treatment response (BASDAI50)

Results: Of the 508 enrolled pts (females: $46.7 \%$, age: $41.4+11.6$ years old, HLA B27 positive: $57.5 \%$, X-Ray sacroiliitis: $53.2 \%$, inflammation at MRI-SIJ: $52.0 \%$ ), only 59 had no structural damage at SIJ-X-Rays and no inflammation at MRI-SIJ (12\%). A concomitant FM was noticed in $42 \%$ vs $37 \%$ of pts treated in accordance vs not with the EMA recommendation, respectively $(\mathrm{p}=0.44)$. The anti-TNF treatment effect was higher in the groups of pts treated in accordance (vs not) with the EMA recommendation (BASDAI 50: $52 \%$ vs 39\%, respectively; $\mathrm{p}=0.054$ ). There was a trend (but not statistically significant) suggesting an interaction of the concomitant FM on the anti TNF treatment response with regard 
to the application of the EMA recommendation (interaction $p$ value $=0.140$ ). In the 59 pts not treated in accordance with the EMA recommendation, the treatment effect in the sub-groups of pts without (vs with) concomitant FM was $38 \%$ vs $40 \%$ respectively, $\mathrm{p}=0.891$. In the 449 pts treated in accordance with the EMA recommendation, the treatment effect in the sub-groups of pts without (vs with) concomitant FM was $56 \%$ vs $46 \%$, $p=0.042$ ).

Conclusions: This study suggests that $1 /$ French rheumatologists are applying the EMA recommendation in daily practice 2/ these recommendations result in a better outcome in terms of short term symptomatic treatment effect. In this study, concomitant FM was not more frequently observed in patients without ( $v S$ with) objective sign of structural damage or inflammation and the impact of a concomitant FM was not more pronounced (or even lower) in pts without (vs with) objective sign of structural damage or inflammation.

\section{References:}

[1] Perrot S, et al. Development and validation of the Fibromyalgia Rapid Screening Tool (FiRST). Pain.2010;150:250-6.

Acknowledgements: This study was conducted thanks to an unrestricted grant from MSD.

Disclosure of Interest: None declared

DOI: 10.1136/annrheumdis-2017-eular.4718

\section{\begin{tabular}{|l|l}
\hline THU0358 ROSUVASTATIN IMPROVES NITRIC OXIDE AND \\
\hline
\end{tabular} ENDOTHELIAL FUNCTION AND SUPPRESSES INFLAMMATORY DISEASE ACTIVITY IN ANKYLOSING SPONDYLITIS}

A. Syngle ${ }^{1}$, N. Garg ${ }^{2}$, P. Krishan ${ }^{2} .{ }^{1}$ Cardio Rheuma, Healing Touch City Clinic, Chandigarh and Fortis Multi Speciality Hospital, Mohali, India, Chandigarh; ${ }^{2}$ Department of Pharmaceutical Sciences and Drug Research, Punjabi University, Patiala, India

Background: Nitric oxide (NO) regulates the synthesis of several inflammatory mediators, functions of inflammatory cells in the inflamed joint and plays a central role in the regulation of blood vessel tone ${ }^{1}$. Therefore, NO inhibitors represent important therapeutic advancement in the management of inflammatory diseases. Rosuvastatin improves $\mathrm{NO}$ and endothelial dysfunction in patients with heart failure ${ }^{2}$ but its effect on NO has not yet been tested in Ankylosing Spondylitis (AS) patients.

Objectives: To investigate the effect of rosuvastatin on nitrite levels (NO surrogate) and its relationship with endothelial function and inflammatory measures in AS.

Methods: 40 consecutive patients (20 in Rosuvastatin (10 mg/day) and 20 in placebo arm) meeting the modified New York criteria for AS, with active disease despite treatment with conventional synthetic DMARDs were recruited. Serum nitrite estimation was carried out by Griess reaction. Flow-mediated dilatation (FMD) was assessed using AngioDefender. Inflammatory measures includedBASDAI, BASFI, ESR and CRP. Pro-inflammatory cytokines (TNF- $\alpha$, IL- 6 and IL-1) were measured at baseline and after 24 weeks.

Results: After 24 weeks, significant improvement in serum nitrite was observed in rosuvastatin group $(5.27 \pm 0.26$ to $4.11 \pm 0.19, \mathrm{p}<0.01)$ compared with placebo $(5.47 \pm 0.26$ to $5.36 \pm 0.23, \mathrm{p}=0.33$ ). At 24 weeks; FMD, TNF- $\alpha$, and IL-6 improved significantly in rosuvastatin group compared with placebo. At 24 weeks; ESR, CRP, BASDAI and BASFI significantly improved in rosuvastatin group compared with placebo. After treatment with rosuvastatin, nitrite correlated inversely with FMD $(r=-0.47, p=0.03)$ (Fig.1A) and positively with TNF- $\alpha(r=0.64, p=0.01)$ (Fig.1B), CRP ( $r=0.52, p=0.01$ ) (Fig.1C) and LDL ( $r=0.54, p=0.01$ ) (Fig.1D).

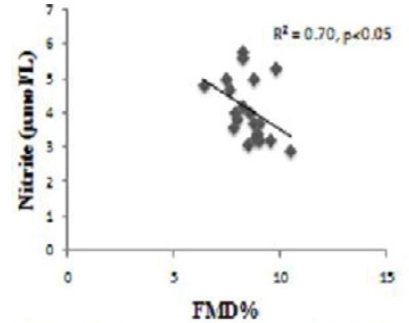

Fig.1A Correlation of nitrite with FMD after treatment with rosuvastatin

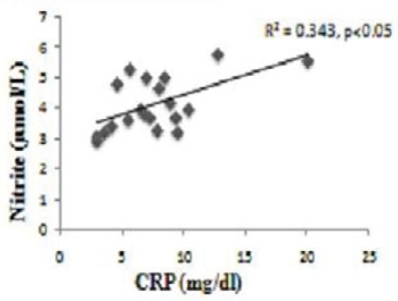

Fig,1C Correlation of nitrite with CRP after Fig treatment with fo survastatin

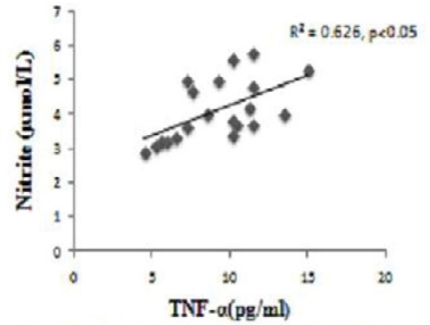

Fig.lB Correlation of nitrite with TNF-a after treatment with rosuvastatin

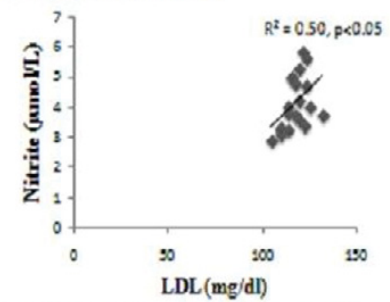

Fig.1D Cocrelation of nitrite with LDL after treatment with roswastatin
Conclusions: Rosuvastatin reduced serum nitrite concentration and improved endothelial dysfunction in AS patients. Rosuvastatin lowers the proinflammatory cytokines, especially IL- 6 and TNF- $\alpha$, which downregulates CRP production and thus the production of NO. Rosuvastatin also favorably improved the lipid levels in AS patients. Rosuvastatin exerts anti-inflammatory, immunomodulatory and vasculoprotective effect in ankylosing spondylitis through both cholesterol dependent and cholesterol independent pathways

References:

[1] Sharma et al. Inflammopharmacology 2007;15:252-9.

[2] Schäfer et al. Arterioscler Thromb Vasc Biol 2005;25:1071-1077.

Acknowledgements: None.

Disclosure of Interest: None declared

DOI: 10.1136/annrheumdis-2017-eular.5062

\section{THU0359 SECUKINUMAB DEMONSTRATES CONSISTENT SAFETY OVER LONG-TERM EXPOSURE (UP TO 3 YEARS) IN PATIENTS WITH ACTIVE ANKYLOSING SPONDYLITIS: POOLED ANALYSIS OF THREE PHASE 3 TRIALS}

A. Deodhar ${ }^{1}$, X. Baraliakos ${ }^{2}$, H. Marzo-Ortega ${ }^{3}$, J. Sieper ${ }^{4}$, M. Andersson ${ }^{5}$, B. Porter ${ }^{6}$, T. Fox ${ }^{5}$. ${ }^{1}$ Oregon Health \& Science University, Portland, United States; ${ }^{2}$ Rheumazentrum Ruhrgebiet Herne, Ruhr-University Bochum, Herne, Germany; ${ }^{3}$ Nihr Lmbru, Ltht\&lirmm, UoL, Leeds, United Kingdom; ${ }^{4}$ Charité University Medicine Berlin, Berlin, Germany; ${ }^{5}$ Novartis Pharma AG, Basel, Switzerland; ${ }^{6}$ Novartis Pharmaceuticals Corp., East Hanover, United States

Background: Safety data for secukinumab in the treatment of ankylosing spondylitis (AS) have been reported from three Phase 3 studies: MEASURE 1 (NCT01358175) $^{1}$, MEASURE 2 (NCT01649375) $^{1}$ and MEASURE 3 (NCT02008916). ${ }^{2}$

Objectives: To report long-term (up to 3 years) pooled safety and tolerability data for secukinumab in AS (data cut-off: 25 June 2016).

Methods: Overall, 371, 219 and 226 patients with active AS were randomised in MEASURE 1, MEASURE 2 and MEASURE 3, respectively. Study design, efficacy and safety results of these studies have been published earlier. ${ }^{1,2}$ Secukinumab doses differed in the studies and included intravenous $10 \mathrm{mg} / \mathrm{kg}$ or subcutaneous (75-300mg) multi-dose loading, followed by subcutaneous (s.c.) maintenance dosing $(75,150$, or $300 \mathrm{mg})$. Data collected up to the last patient performing the Wk 156 visit in MEASURE 1, the Wk 104 visit in MEASURE 2, and the Wk 52 visit in MEASURE 3 were pooled at the patient level. Exposure-adjusted incidence rates were calculated to account for differences in treatment exposure and analyses included all patients who received $\geq 1$ dose of secukinumab 150 or 300mg.

Results: A total of 510 patients were included in the analysis ( 968.9 patient-years of exposure). The exposure-adjusted $A E$ and SAE rates with secukinumab across the entire safety period were 159.2 and 5.4 per 100 patient-years, respectively. Nasopharyngitis, diarrhoea and headache were the most frequently reported AEs. The incidences of Candida infections, serious infections, inflammatory bowel disease, major adverse cardiac events, neutropenia and uveitis were low and consistent with previous reports over shorter exposure periods ${ }^{1}$ (Table). No cases of suicidal ideation or depression were reported.

Table 1. Summary of pooled safety across 3 AS studies (Entire safety period)

\begin{tabular}{lc}
\hline & Any secukinumab dose $(\mathrm{N}=510)$ \\
\hline Total exposure, patient-years & 968.9 \\
Minimum-maximum exposure (days) & $1-1530$ \\
Death, $\mathrm{n}(\%) \quad$ AEs by EAIR: AE per 100 Patient-years $(95 \% \mathrm{Cl})$ & $159.2(144.4,175.1)$ \\
& $5.4(4.0,7.1)$ \\
Any AE & \\
Any SAE & $13.6(11.2,16.5)$ \\
Frequent AEs ${ }^{1}$ & $6.4(4.9,8.3)$ \\
$\quad$ Nasopharyngitis & $6.7(5.1,8.7)$ \\
Diarrhoea & $4.3(3.1,5.9)$ \\
Headache & \\
Upper respiratory tract infection & $0.8(0.4,1.6)$ \\
AEs of special interest & $0.7(0.3,1.5)$ \\
$\quad$ Candida infections & $0.4(0.1,1.1)$ \\
Serious infections & $0.2(0.0,0.7)$ \\
Inflammatory Bowel Disease & $0.2(0.0,0.7)$ \\
Crohn's disease & $0.4(0.1,1.1)$ \\
Ulcerative colitis & $1.2(0.6,2.1)$ \\
MACE & $1.6(0.9,2.6)$ \\
Neutropenia &
\end{tabular}

${ }^{1} \mathrm{AEs}$ that occurred in Any secukinumab group with an $\mathrm{IR}>4.0$ during the entire safety period. $\mathrm{AE}$ adverse event; $\mathrm{Cl}$, confidence interval; EAIR, exposure adjusted incidence rate per 100 patientyears; MACE, major adverse cardiac events; $\mathrm{N}$, number of patients in the analysis; $n$, number of patients with event; SAE, serious adverse event.

Conclusions: This longer-term safety assessment of secukinumab in the treatment of AS was consistent with previous reports and did not identify any new safety signals.

References:

[1] Baeten D, et al. N Engl J Med 2015;373:2534-48.

[2] Kivitz A, et al. XIX PANLAR 2016, Panamá City, Panama. Poster No. P-081. Disclosure of Interest: A. Deodhar Grant/research support from: AbbVie, Amgen, 